\title{
TOPIC: PUBIC INGUINAL PAIN SYNDROME (PIPS) - Sportsman
}

\author{
VS:55 \\ HERNIA OBSTURATORIA AS ANOTHER ENTITY \\ OF PATIENTS WITH THE SO-CALLED \\ SPORTMAN HERNIA: THE IMPORTANT ROLE \\ OF LAPAROSCOPIC APPROACH \\ S Morales-Conde ${ }^{1}$, M Sanchez-Ramirez ${ }^{2}$, I Alarcón' ${ }^{1}$ A Barranco', \\ J Gómez-Menchero ${ }^{2}$, J M Suárez ${ }^{2}$, J Bellido², M Socas \\ ${ }^{1}$ Hospital Universitario Virgen del Rocio, Sevilla, Spain \\ ${ }^{2}$ Hospital Quirón-Sagrado Corazón, Sevilla, Spain
}

Introduction: The hernia obturatoria in an extremely rare entity (less than $1 \%$ of hernias) difficult to diagnose since these hernias do not show any specific signs and symptoms, being possible the preoperative diagnose in just $10-30 \%$ of the cases. The delay of the diagnose is also related to adelay in the treatment what could be realted to a high incidence of emergency situations as strangulation.

On the other hand, the so-called sportman hernia is an entity still to be defined properly, being included different entities as responsible of the inguinal pain, being described in very few cases the hernia obturatoria as a source of this syndrome.

Patient and method: A 19 years old male, non-professional athletic but very active practicing soccer was attended showing and left inguinal pain while practicing sport. An ultrasound did not show the present

\begin{abstract}
of an inguinal hernia nether a deficiency of the posterior inguinal wall. Clinical examination only detected a dilation of the external inguinal ring. The patient was study by the orthopedic surgeons establishing a relationship between the inguinal pain and an enthesitis of the abductor. A conservative treatment together with physiotherapy was conducted with no improve of the inguinal pain. Since the sumptoms showed by the patient did not allow to perform any sport a preperitoneal endoscopic (TEP) approach of the left inguinal hernia was proposed. A hernia obturatoria was found during the surgery after identifying the nerve and artery obturatoria, founding a lipoma including in the hernia defect compressing the nerve as a potential cause of the inguinal pain. The lipoma was reduced and a light weight PP mesh was placed being performed an atraumatic fixation with fibrin glue.
\end{abstract}

Results: Total surgical time was 18 minutes, being the patient discharged form the hospital 14 hours later. Patient re-started full physical activity 3 weeks after surgery being the follow-up of the patient of 14 month with no recurrence of the pain.

Conclusion: Total endoscopic preperitoneal apporach (TEP) of the iguinal región is a safe alternative to patient with a hernia obturatoria. These hernias should be considered as a source of inguinal pain in patients with the so-called sportman hernia, being very difficult to be diagnosed. When no other source of pain is found a TEP should be consider to detect this type of entities. 